\title{
Método de valoración de la viabilidad de corredores biológicos para mamíferos: análisis preliminar del corredor biológico San Juan-La Selva en Costa Rica
}

\author{
Valuation method to evaluate the viability of biological corridors for \\ mammals: a preliminary analysis of \\ San Juan-La Selva corridor in Costa Rica
}

\author{
José F. González-Maya ${ }^{1,2}$, I. Mauricio Vela-Vargas ${ }^{1}$, Alexandra Pineda-Guerrero ${ }^{1}$ \\ Resumen
}

\begin{abstract}
Los corredores biológicos constituyen una estrategia fundamental en la conservación de la biodiversidad en paisajes fragmentados. Sin embargo, existen pocas evidencias y aproximaciones acerca de su funcionalidad o la viabilidad de sus poblaciones a largo plazo en especial en el trópico. Se realizó una aproximación teórico-descriptiva para determinar el riesgo de extinción de diferentes especies de mamíferos, basado en tres parámetros principales: vulnerabilidad de la especie, requerimientos de área y requerimientos de hábitat, siendo cada una de estas categorías calificadas en los niveles de alto, medio y bajo. Para los mamíferos grandes hay una alta probabilidad de extinción, mientras que para los mamíferos medianos y pequeños, la probabilidad de extinción es baja y una alta probabilidad de mantenimiento de poblaciones viables con una alta disponibilidad de hábitat.
\end{abstract}

Palabras clave: Corredor, Costa Rica, Mamíferos, Riesgo de extinción.

\begin{abstract}
Biological corridors are fundamental strategies for biodiversity conservation in fragmented landscapes. However, few evidence exist respecting their functionality or the population viability of its species on long term especially for the tropics. We performed a theoretical-descriptive approach to determine mammals' risk of extinction based on three main parameters: species vulnerability and area and habitat requirements, each one of these categories were classified in levels of high, medium and low. For large mammals there is a high probability of extinction, while for medium and small mammals there is a low probability of extinction and a high probability of permanence of viable populations with high habitat availability.
\end{abstract}

Keywords: Corridor, Costa Rica, Extinction risk, Mammals.

\section{Introducción}

Históricamente, la conservación de especies y ecosistemas se ha enfocado en la creación y mantenimiento de áreas protegidas con el fin de salvaguardar obligatoriamente grandes áreas de ecosistemas naturales y los organismos que sobreviven dentro de estos (Gardner et al. 2009, Chazdon et al. 2009, González-Maya et al. 2010). A pesar de la importan- cia fundamental de las áreas protegidas para la conservación de la biodiversidad, se reconoce que no es la única opción para asegurar la perdurabilidad de los atributos de la biodiversidad, por lo que el reconocimiento de la importancia de los paisajes modificados por las actividades humanas ha sido tomado en cuenta para el establecimiento de planes de manejo y conservación (Putz et al. 2001). Sin embargo, son escasos los esfuerzos de planificación a nivel de

1 Proyecto de Conservación de Aguas y Tierras, ProCAT Colombia and Sierra to Sea Institute. Las Alturas, Coto Brus, Puntarenas, Costa Rica. e-mail: jfgonzalezmaya@gmail.com

2 Instituto de Ecología, Universidad Nacional Autónoma de México, 04510, México DF, México.

Fecha recepción: Febrero 19, 2013

Fecha aprobación: Abril 18, 2013

(c) Rev. Biodivers. Neotrop. 2013; 3 (1): 30-6 
paisaje, necesarios para predecir y prevenir los impactos sobre poblaciones de fauna silvestre, sobre todo en el trópico (Chazdon et al. 2009). Adicionalmente, las dificultades intrínsecas, logísticas y económicas, para el desarrollo de estudios ecológicos sistemáticos en el trópico y la poca disponibilidad de información tanto espacial a resolución adecuada como ecológica de la mayoría de especies tropicales, hacen que los esfuerzos de planificación sean aún más complejos (Cuarón 2000).

Los corredores biológicos han sido utilizados como una de las principales estrategias de restauración de la dinámica de los paisajes, ya que estos incrementan la conectividad, favoreciendo un incremento en los tamaños poblacionales, así como el intercambio de genes en áreas que han sufrido perturbaciones en la continuidad de sus hábitat (Baum et al. 2004). A pesar de la utilidad probada de este tipo de iniciativas, muchos corredores aún carecen de evaluaciones a la escala y dimensión biológica adecuadas, y estos han sido principalmente diseñados y establecidos a partir de perspectivas estructurales del paisaje y no de la respuesta de los organismos frente a los cambios en el mismo (Baguette et al. 2013). Además, en muchos casos la definición de los corredores ha sido un proceso más de tipo político y en muchos otros aún carecen de información o validación biológica (Forman 1991). Por las limitantes de información para la validación de corredores y la necesidad de contar con herramientas que permitan la planificación de paisajes, el objetivo del presente trabajo fue diseñar una herramienta de valoración teórico-descriptiva que permita evaluar la viabilidad del Corredor Biológico San Juan-La Selva en Costa Rica para diferentes especies de mamíferos, representando el rango de variación en biomasa, basado en información teórica y como aproximación preliminar como insumo para la toma de decisiones.

\section{Metodología}

El Corredor Biológico San Juan-La Selva (CBSJLS) se encuentra ubicado en la zona norte de Costa Rica, en las provincias de Heredia y Alajuela (Chassot y Monge 2002), cubre un área aproximada de $2473.05 \mathrm{~km}^{2}$, de los cuales casi $64 \%$ corresponden a bosque (Ramos 2004; Figura 1). Su rango altitudinal se encuentra entre los 30 y los $3000 \mathrm{msnm}$, en las que se han documentado al menos ocho zonas de vida, entre las que destacan el bosque muy húmedo premontano y el bosque muy húmedo tropical (Holdridge 1978, Chassot y Monge 2002). Este co-

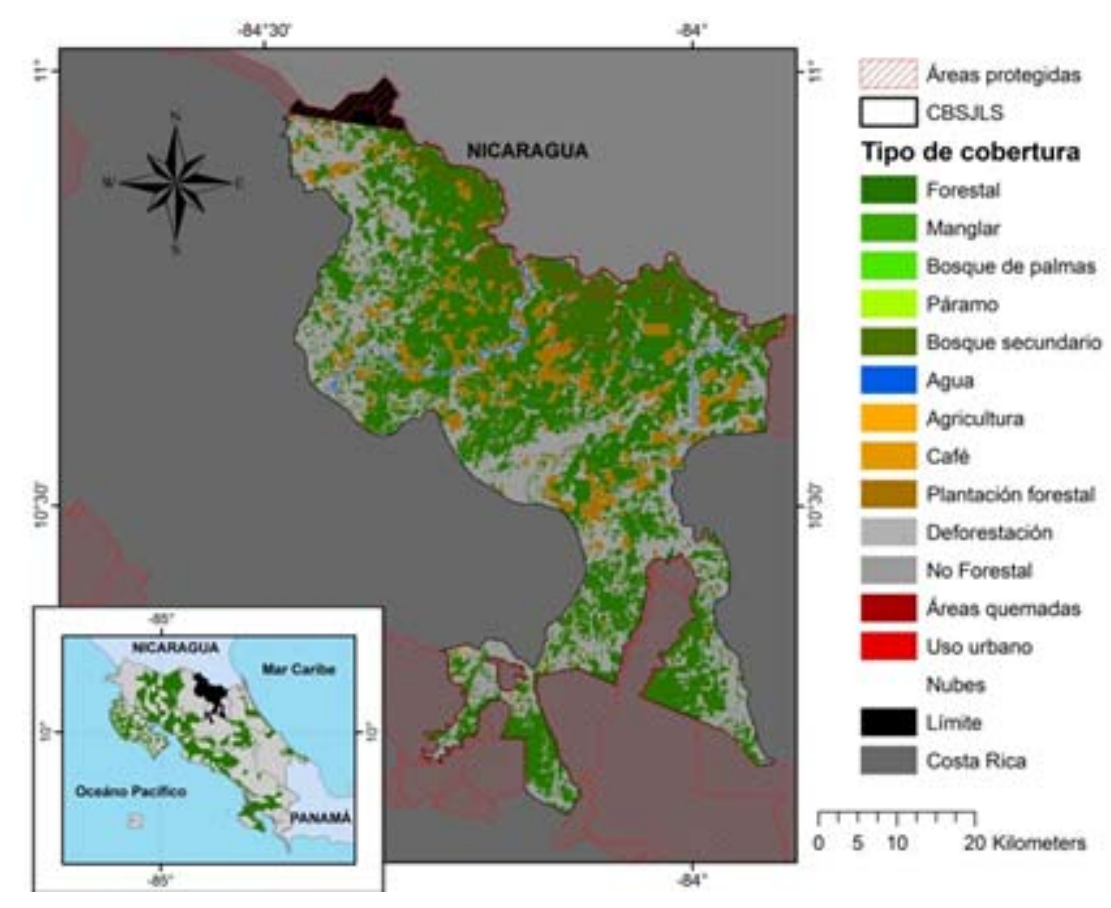

Figura 1. Ubicación y coberturas del Corredor Biológico San Juan-La Selva en Costa Rica. 
rredor se caracteriza por tener tres tipos de bosque: bosques de Pentaclethra macroloba, bosques de Qualea paraensis, Vochysia ferruginea y Couma macrocarpa y bosques dominados por Pentaclthra macroloba y Carapa guianensis.

El CBSJLS se considera una de las iniciativas de conectividad pioneras en Costa Rica y es reconocida por el mejoramiento y mantenimiento de sus coberturas naturales y al aumento de los procesos de conectividad que brinda, lo cual permite el mantenimiento de las poblaciones de flora y fauna silvestre a largo plazo y los servicios ecosistémicos que están presta a las comunidades humanas (Noss 1983, Rosenberg et al. 1997).

Con el fin de evaluar de forma preliminar la viabilidad del corredor para mamíferos, se construyó una aproximación teórica para determinar cuál es el riesgo de extinción de diferentes especies de mamíferos, basado en tres parámetros principales: vulnerabilidad de la especie, requerimientos de área, requerimientos de hábitat y capacidad de dispersión (Daily et al. 2003, Cardillo et al. 2008). Para cada categoría se elaboró una tabla de calificación con tres niveles alto, medio y bajo las cuales representan un puntaje de 3, 2 y 1 respectivamente (Tabla 1 ).

A pesar de que el tamaño mínimo viable poblacional está determinado por factores intrínsecos de las especies, factores ambientales y son en general específicos de cada sitio por la naturaleza teórica y preliminar de esta aproximación, se utilizaron tamaños poblacionales derivados de datos reportados en la literatura (Traill et al. 2007). Se utilizó un cálculo aproximado del tamaño poblacional mínimo viable estandarizado derivado de los valores reportados por Traill et al. (2007), estimado como el promedio de los tamaños de población mínimos viables estandarizadas (límite superior del intervalo de confianza del 95\%) estimadas de acuerdo con el tamaño corporal y grupo taxonómico (Traill et al. 2007). En el caso de que existiesen estimaciones específicas para la especie, se usó el dato directo de tamaño poblacional mínimo viable estandarizado. A pesar de que los tamaños poblacionales mínimos varían entre especies similares y dependiendo de los hábitats, la no existencia de valores específicos y el uso de los límites superiores estandarizados de especies similares provee de una medida conservadora de tamaño mínimo poblacional (Traill et al. 2007). El cálculo de requerimiento de área se basó en el promedio de hábitat interior para el corredor (Ramos 2004), dividido entre tamaño poblacional viable mínimo para cada especie (Frankham 1995). Luego se realizó la suma por especie obteniendo una clasificación global, la cual representa una categoría de viabilidad para diferentes especies de diferentes tamaños en el corredor (Tabla. 2).

Con información de historia natural de especies de pequeños (Peromyscus mexicanus, Nyctomys sumichrasti), medianos (Alouatta paliata, Potos flavus) y grandes mamíferos (Panthera onca, Tapirus baiirdi) presentes en el CBSJLS (Tabla 3), se calculó la viabilidad teórica de las especies. La información usada se tomó principalmente de la base de datos PanTheria (Jones et al. 2009) así como estudios de campo (Schaller y Crawshaw 1980, Ford y Hoffmann 1988, Zucker et al. 1996, Foerster 1998,

Tabla 1. Categorías y criterios de clasificación para el análisis preliminar de viabilidad de poblaciones de mamíferos en el Corredor Biológico San Juan-La Selva, Costa Rica

\begin{tabular}{|c|c|c|c|}
\hline Categoría/Calificación & Alto $(3)^{*}$ & Medio (2) & Bajo (1) \\
\hline Vulnerabilidad específica & $\begin{array}{l}\text { Poblaciones reducidas, grado } \\
\text { de amenaza, presión de } \\
\text { cacería }\end{array}$ & Menor grado de amenaza & $\begin{array}{l}\text { Grado de amenaza } \\
\text { mínimo }\end{array}$ \\
\hline Requerimiento de área & $\begin{array}{l}\text { Ámbito de hogar y requerimien- } \\
\text { tos de hábitat mayores a la } \\
\text { capacidad de carga }\end{array}$ & $\begin{array}{l}\text { Igual a la capacidad de } \\
\text { carga }\end{array}$ & $\begin{array}{l}\text { Menor a la capacidad } \\
\text { de carga }\end{array}$ \\
\hline Hábitat y dispersión & $\begin{array}{l}\text { Especialista, hábitat restringido, } \\
\text { y baja movilidad }\end{array}$ & $\begin{array}{l}\text { Requerimientos moderados } \\
\text { y movilidad moderada }\end{array}$ & $\begin{array}{l}\text { Requerimientos } \\
\text { bajos }\end{array}$ \\
\hline
\end{tabular}

*El número () indica la calificación 
Daily et al. 2003). Los datos de densidad se estimaron a partir del promedio de los datos reportados en la literatura para hábitats similares (Schaller y Crawshaw 1980, Ford y Hoffmann 1988, Eisenberg 1989, Naranjo 1995, Foerster 1998, Hunt et al. 2004, Salom et al. 2007, González-Maya et al. 2008, Schnell et al. 2010, Trujano-Alvarez y AlvarezCastañeda 2010, González-Maya et al. 2013).

\section{Resultados}

La viabilidad de las poblaciones de mamíferos mostró un valor muy diferenciado dependiendo sobre todo de los requerimientos de área (Tabla 3). Sin embargo, dadas algunas características intrínsecas de las especies, independientemente del tamaño, la viabilidad puede variar entre una y otra especie. Para los mamíferos pequeños, $P$. mexicanus, considerada generalista (Reid y Pino 2008), y N. sumichrasti, especie netamente arborícola (Samudio et al. 2008), presentan un bajo grado de amenaza variando muy poco en términos de su viabilidad potencial, siendo menos limitados por la amplia disponibilidad de hábitat. Las especies medianas, P. flavus y A. palliata, a pesar de no variar en términos de hábitat disponible, presentan una diferenciación notable en cuanto a su viabilidad, por su vulnerabilidad y requerimientos de hábitat. Por su parte las especies de mayor tamaño se encuentran fuertemente amenazadas en el corredor, y presentan una muy baja viabilidad a largo plazo; estas especies se ven muy afectadas tanto por su vulnerabilidad intrínseca como por sus requerimientos de área (Tabla 4).

En general, el aspecto cuantitativo de esta aproximación muestra sobre todo las limitantes de hábitat disponible para especies con requerimientos amplios. Se observó que para especies de gran tamaño como el jaguar ( $P$. onca) no existe área suficiente para mantener poblaciones viables en todo el corredor, y menos para las áreas de bosque $(2.5 \%$ de la pobla-

Tabla 2. Calificación global y categoría de probabilidad de extinción local de poblaciones de mamíferos en el Corredor Biológico San Juan-La Selva, Costa Rica

\begin{tabular}{ccl}
\hline Categoría & Calificación & \multicolumn{1}{c}{ Categoría } \\
\hline 1 & $3-5$ & Baja probabilidad de extinción local \\
2 & $5-7$ & Moderada probabilidad de extinción local \\
3 & $7-9$ & Extinto/Alta probabilidad de extinción local \\
\hline
\end{tabular}

Tabla 3. Aspectos de historia natural, capacidad de carga del corredor y población potencial de mamíferos pequeños, medianos y grandes para la evaluación del Corredor Biológico San Juan-La Selva, Costa Rica

\begin{tabular}{|c|c|c|c|c|c|c|c|}
\hline Grupo & Tamaño & Especie & $\begin{array}{l}\text { Tamaño } \\
\text { PMVst }^{\mathrm{a}}\end{array}$ & $\begin{array}{l}\text { Densidad } \\
\text { s promedio } \\
\left(\text { ind } / \mathrm{km}^{2} \text { ) }\right.\end{array}$ & $\begin{array}{l}\text { Área } \\
\text { requeridab }\end{array}$ & $\begin{array}{l}\text { Capacidad } \\
\text { de carga }^{c}\end{array}$ & $\begin{array}{l}\% \text { población } \\
\text { potenciald }^{\mathrm{d}}\end{array}$ \\
\hline Carnívoro & Grande & Panthera onca & 3.876 & 0,06 & $64.600,00$ & 95,37 & 2,46 \\
\hline Herbívoro & & Tapirus bairdii & 6.832 & 0,70 & $9.760,00$ & $1.112,67$ & 16,29 \\
\hline $\begin{array}{l}\text { Frugívoro/ } \\
\text { Folívoro }\end{array}$ & Mediano & Alouatta palliata & 3.916 & 54,75 & 71,53 & $87.026,77$ & $2.222,34$ \\
\hline \multirow[t]{3}{*}{ Omnívoro } & Pequeño & $\begin{array}{l}\text { Potos flavus } \\
\text { Peromyscus }\end{array}$ & 3.046 & 17,16 & 177,51 & $27.276,33$ & 895,48 \\
\hline & & $\begin{array}{l}\text { mexicanus } \\
\text { Nyctomys }\end{array}$ & 6.149 & 78,00 & 78,83 & $123.983,34$ & $2.016,32$ \\
\hline & & sumichrasti & 4.506 & 87,00 & 51,79 & $138.289,11$ & $3.069,00$ \\
\hline
\end{tabular}

\footnotetext{
a Población mínima viable estandarizada de acuerdo a Traill et al. (2007)

b Área requerida de hábitat para soportar una población mínima viable

${ }^{c}$ Estimada como el número de individuos potenciales según densidad y disponibilidad de hábitat en el corredor (1589.53 km²)

a Porcentaje de la población potencial en el corredor con respecto a la población mínima viable
} 
Tabla 4. Ejemplo de aplicación de la calificación por gremio y categoría final de poblaciones de especies de mamíferos en el Corredor Biológico San Juan-La Selva, Costa Rica

\begin{tabular}{llccccc}
\hline Grupo & Especie & Vulnerabilidad & Área & Hábitat & Calificación & Categoría \\
\hline Carnívoro & Panthera onca & $\mathrm{A}$ & $\mathrm{A}$ & $\mathrm{M}$ & 8 & 3 \\
Herbívoro & Tapirus bairdii & $\mathrm{A}$ & $\mathrm{A}$ & $\mathrm{M}$ & 8 & 3 \\
\multirow{2}{*}{ Omnívoro } & Peromyscus mexicanus & $\mathrm{B}$ & $\mathrm{B}$ & $\mathrm{B}$ & 3 & 1 \\
\multirow{2}{*}{ Frugívoro/Folívoro } & Nyctomys sumichrasti & $\mathrm{B}$ & $\mathrm{B}$ & $\mathrm{M}$ & 4 & 1 \\
& Potos flavus & $\mathrm{A}$ & $\mathrm{B}$ & $\mathrm{M}$ & 6 & 2 \\
& Alouatta palliata & $\mathrm{M}$ & $\mathrm{B}$ & $\mathrm{B}$ & 4 & 1 \\
\hline
\end{tabular}

ción mínima viable considerando homogeneidad de hábitats), siendo ligeramente mayor la población potencial de danta (T. bairdii) aunque esta no alcanza la población mínima viable predicha para la especie. Para mamíferos medianos y pequeños, en general hay una alta o mediana probabilidad de mantenimiento de poblaciones viables con una alta disponibilidad de hábitat.

\section{Discusión}

El mantenimiento de especies de mamíferos a largo plazo depende de múltiples aspectos como lo son la biología, ecología e historia natural de las especies, además de los factores extrínsecos que ejercen presión y amenazan sus poblaciones. La inclusión de características de las especies como el área de hábitat disponible, la evaluación de las matrices de paisaje, el ámbito de hogar de las especies y la dieta, permiten la evaluación real del estado y las probabilidades de extinción de las especies, con el fin de crear corredores funcionales con enfoques multiespecíficos (Fahrig y Merriam 1994, Lindenmayer y Henry 1993, Baum et al. 2004).

Dentro del CBSJLS, las altas probabilidades de extinción para los mamíferos grandes se puede atribuir a la baja disponibilidad de hábitat y a las bajas densidades de los mismos, además del aislamiento de parches de bosque, factores que llevan a la pérdida de poblaciones locales y luego a la pérdida de estas especies dentro del paisaje (Baguette et al. 2013, Jorge et al. 2013). En el caso de los mamíferos pequeños y medianos, a pesar de que en términos de área pueden existir condiciones suficientes y que las especies evaluadas parecen no ser tan altamente susceptibles, es necesario evaluar otros aspectos de su ecología y amenazas para asegurar su per- manencia en el paisaje; factores de índole metapoblacional y genético y de presiones por fragmentación y cacería, entre otros, se deben evaluar directamente para confirmar esta viabilidad. Existen además otros aspectos que pueden variar la respuesta de las especies a las presiones, como la plasticidad poblacional como respuesta a reducciones o pérdida de hábitat, que pueden cambiar la tendencia en especial para las especies pequeñas, sin embargo, en términos conservativos, la viabilidad de una población dadas amenazas constantes y pérdida de hábitat deberían ser consideradas como más probables que el efecto opuesto.

A pesar de las diferentes presiones que lo afectan, el CBSJLS todavía presenta una cobertura de hábitats de bosque significativa y una conectividad entre sus parches que aportan significativamente a la conservación de poblaciones de mamíferos (Canet 2010). A su vez, existen reportes recientes incluso de las especies grandes en el corredor, lo que indican que aún hay algunas condiciones para su permanencia (Chassot et al. 2008), sin esto significa que estas sean viables al largo plazo. A su vez, por las restricciones de área y las constantes amenazas por monocultivos, extracción de madera y cacería, es necesario continuar con las acciones que permitan el mantenimiento de diferentes grupos de especies, aumenten la permeabilidad al movimiento de individuos dentro del CBSJLS (Baum et al. 2004, Gardner et al. 2009), y así mantener las funciones ecosistémicas y los servicios ambientales del territorio a nivel regional. El manejo de presiones como el aumento de la frontera agrícola y ganadera, y el adecuado manejo de especies domésticas, por el posible conflicto con las especies silvestres, son aspectos que deben ser priorizados en las agendas de políticas y gestión de las autoridades ambientales (Jorge 
et al. 2013). El CBSJLS ha realizado un importante avance a lo largo de los últimos 10 años, por lo que es reconocido como uno de los corredores biológicos pioneros a nivel nacional y a nivel Mesoamericano (Canet 2010). No obstante, se deben buscar e incentivar alternativas de producción sostenible que beneficien a los habitantes de la zona y que permitan seguir generando los procesos de conectividad y de esta forma mayor viabilidad y funcionabilidad del corredor por la alta probabilidad de extinción, en especial de grandes mamíferos a los que está expuesto.

La aproximación propuesta sobresimplifica el análisis de viabilidad de poblaciones o funcionalidad de un corredor, pero evidencia la necesidad de enfocar acciones en especial en el aumento de las áreas de hábitat efectivas y la conectividad en el marco de paisajes tropicales fragmentados. Las herramientas de modelación tanto espacial (ocupación, modelos de distribución de especies entre otros), como de viabilidad poblacional junto con las herramientas genéticas, pueden proveer de evidencias certeras acerca de la funcionalidad e importancia de los corredores para las poblaciones de mamíferos.

\section{Agradecimientos}

El presente trabajo fue realizado gracias a CATIE, The Sierra to Sea Institute y The Mikelberg Family Foundation. Un especial agradecimiento a Andrés Arias-Alzate por la revisión del manuscrito. A los revisores y el editor por su aporte significativo al mejoramiento del manuscrito.

\section{Literatura Citada}

Baguette M, Mennechez G. 2004. Resource and habitat patches, landscape ecology and metapopulation biology: a consensual point. Oikos. 106 (2): 399-403.

Baguette M, Blanchet S, Legrand S, Stevens VM, Turlure C. 2013. Individual dispersal, landscape connectivity and ecological networks. Biol Rev Cambridge Philosoph Soc. 88: $310-26$.

Baum KA, Haynes KJ, Dillemuth FP, Cronin JT. 2004. The matrix enhances the effectiveness of corridors and stepping stones. Ecology. 85: 2671-6.

Canet L. 2010. Diagnóstico sobre la efectividad de manejo de los corredores biológicos de Costa Rica. Informe Fase I. Turrialba: Centro Agronómico Tropical de Investigación y Enseñanza (CATIE). 382 pp.
Cardillo M, Mace GM, Gittleman JL, Jones KE, Bielby J, Purvis A. 2008. The predictability of extinction: biological and external correlates of decline in mammals. Proceed Biol Sci Royal Soc. 1641 (275): 1441-8.

Chassot O, Monge G. 2002. Corredor Biológico San Juan-La Selva: Ficha Técnica. San José: Comité Ejecutivo Corredor Biológico San Juan-La Selva. 78 pp.

Chassot O, Monge-Arias G, Jiménez V. 2008. Evaluación del hábitat potencial para la Danta Centroamericana (Tapirus bairdii) en el Corredor Biológico San Juan-La Selva, Costa Rica. Rev Geogr Am Central 42: 97-112.

Chazdon RL, Harvey CA, Komar O, et al. 2009. Beyond reserves: A research agenda for conserving biodiversity in human modified tropical landscapes. Biotropica. 41 (2): $142-53$.

Cuarón A. 2000. Effects of land-cover changes on mammals in a neotropical Region: a modeling approach. Conserv Biol. 14 (4): 1676-92.

Daily G, Ceballos G, Pacheco J, Suzan G, Sánchez-Azofeifa A. 2003.Countryside biogeography of neotropical mammals: conservation opportunities in agricultural landscapes of Costa Rica. Conserv Biol. 17 (6): 1814-26.

Fahrig L, Merriam G. 1994. Conservation of fragmented populations. Conserv Biol. 8 (1): 50-9.

Ford LS, Hoffmann RS. Potos flavus. Mamm Spec. 321: 1-9.

Forman, RTT. 1991. Landscape corridors: from theoretical foundations to public policy. Nat Conserv. 2: 71-84.

Hunt JL, Morris JE, Best TL. 2004. Nyctomys sumichrasti. Mamm Spec. 754: 1-6.

Frankham R. 1995. Effective population size/adult population size ratios in wildlife: a review. Genet Res. 66: 95-107.

Foerster C. 1998. Ecología de la danta centroamericana Tapirus bairdii en un bosque húmedo tropical de Costa Rica. Trabajo de grado Maestría. PRMVS. San José: Universidad Nacional de Costa Rica.65 pp.

Gardner T, Barlow J, Chazdon R, et al. 2009. Prospects for tropical forest biodiversity in a human-modified world. Ecol Letters. 12: 561-82.

Holdridge L. 1978. Ecología basada en zonas de vida. San José: IICA.159 pp.

Jones KE, Bielby J, Cardillo M, et al. 2009. PanTHERIA: a species-level database of life history, ecology, and geography of extant and recently extinct mammals. Ecology. 90: 2648.

Jorge ML, Galleti M, Ribeiro MC, Ferraz KM. 2013. Mammal defaunation as surrogate of trophic cascades in a biodiversity hotspot. Biol Conserv. 163: 49-57.

Lindenmayer DB, Nix HA. 1993. Ecological principles for design of wildlife corridors. Conserv Biol. 7 (3): 627-30.

Naranjo E. 1994. Abundancia, uso de hábitat y hábitos de alimentación del tapir (Tapirus bairdii) en un bosque tropical húmedo en Costa Rica. Trabajo de grado Maestria. PRMVS. San José: Universidad Nacional de Costa Rica. 75 pp.

Noss R. 1983. A regional landscape approach to maintain diversity. Bioscience. 33: 700-6.

Putz F, Blatte G, Redford K, Fimbel R, Robinson J. 2001. Tropical forest management and conservation of 
biodiversity: an overview. Conserv Biol. 15 (1): 7-20.

Ramos Z. 2004. Estructura y composición de un paisaje boscoso fragmentado: Herramienta para el diseño de estrategias de conservación de la biodiversidad. Trabajo de grado Maestría. Turrialba: Centro Agronómico Tropical de Investigación y Enseñanza (CATIE). 114 pp.

Reid F, Pino J. 2008. Peromyscus mexicanus. In: IUCN 2013. IUCN Red List of Threatened Species. Version 2013.1. Downloaded on 05 November 2013. URL: www.iucnredlist.org

Redford K, Robinson J. 1991. Park size and the conservation of forest mammals in Latin America. In: Mares M, Schmidly D (eds.). Latin American mammalogy, history, biodiversity, and conservation. Norman: University of Oklahoma.

Rosenberg D, Noon B, Meslow C. 1997. Ecological role of linear conservation areas for maintaining biological diversity. Bioscience. 47 (10): 677-87.

Salom-Pérez R, Carrillo E, Sáenz J, Mora J. 2007. Critical condition of the jaguar Panthera onca population in
Corcovado National Park, Costa Rica. Oryx 41 (1): 516.

Samudio R, Pino J, Reid F. 2008. Nyctomys sumichrasti. In: IUCN 2013. IUCN Red List of Threatened Species. Version 2013.1. Downloaded on 05 November 2013. URL: www.iucnredlist.org

Schnella GD, Abreu Gillanda E, Sánchez-Hernández C, et al. 2010. Microhabitat preferences and spatial distribution of the vesper rat (Nyctomys sumichrasti) in Colima, Mexico. Mamm Biol - Zeitschrift für Säugetierkunde 75 (6): 482-95.

Shaller G, Crawshaw P. 1980. Movement patterns of jaguar. Biotropica. 12 (3): 161-8.

Trujano-Alvarez AL, Alvarez-Castañeda ST. 2010. Peromyscus mexicanus (Rodentia: Cricetidae). Mamm Spec. 42 (1): 111-8.

Zucker E, Clarke M, Glander K, Scott N. 1996. Sizes of home ranges and Howling Monkeys groups at Hacienda La Pacífica, Costa Rica: 1972-1991. Brenesia. 45-6: 153-6. 